nen, daraus einen entsprechenden Itempool abgeleitet und diesen bei einer schriftlichen Befragung getestet. Während es in diesem Beitrag keine Angabe darüber gibt, wie die Stichprobe rekrutiert wurde, haben sich Christoph Klimmt und Peter Vorderer (beide Hannover) selbstkritisch mit ihrem Erhebungsinstrumentarium auseinander gesetzt. Klimmt und Vorderer wollten die Intensität der parasozialen Interaktion zu Computer- und Fernsehfiguren miteinander vergleichen und haben ihre Erhebung in einer größere schriftliche Befragung integriert, die von der Firma veranstaltet wurde, die Lara Croft erschaffen hat.

Man mag einerseits die Flut von Tagungsbänden bedauern und darauf hinweisen, dass das wirklich Wichtige irgendwann als Monographie erscheint oder schon erschienen ist. Andererseits bietet der vorliegende Band dem Nachwuchs eine Publikationsmöglichkeit, gelungenen Abschlussarbeiten ein Forum und zudem einen Einstieg in ein weites Forschungsfeld. Höher sollte man die Messlatte nicht legen.

Michael Meyen

\section{Eike Hebecker}

\section{Die Netzgeneration}

Jugend in der Informationsgesellschaft

Frankfurt: Campus, 2001. - $212 \mathrm{~S}$.

\section{ISBN 3-593-36838-2}

Bei dieser Arbeit handelt es sich um die Veröffentlichung einer Dissertation (Universität Gießen). Es ist eine theoretische Auseinandersetzung mit den gängigen Interpretationen und Bewertungen der „Medien“. Diese „Diskursanalyse" wird in einem umfangreichen theoretischen Teil ausgearbeitet, in dem der Forschungsstand zum Thema Jugend und Medien ebenso berücksichtigt ist wie methodische und begriffliche Überlegungen zum Diskursbegriff. Die folgenden Kapitel zu den Typen der Jugenddiskurse schließen erfreulicherweise die Auseinandersetzung mit der Geschichte der Jugendbilder und Jugenddebatten mit ein. Mit einem Kapitel zum Konzept der Generationen wird dieser umfangreiche theoretische Teil beendet, der mehr als die Hälfte der Untersuchung umfasst. Schließlich werden in den beiden Schlusskapiteln die Ergebnisse präsentiert.
Eingangs grenzt sich der Autor in seiner Untersuchung von der Erwartung ab, dass er Belege für einen „anstehenden Generationswechsels" oder empirische Beweise für die Existenz einer „Netzgeneration“ vorlegt (z. B. an Hand demographischer Daten, Nutzungsstudien oder jugendkultureller Artikulationen). Es geht ihm in dieser Arbeit um eine theoretische Auseinandersetzung mit der Art und Weise, wie sich die „gesellschaftliche Thematisierung aktueller Jugendgenerationen " mit der Debatte über die Medien verbinden: „Der Fokus richtet sich ... auf die Verwendung des Diskurs- und Deutungsmusters, Generation' im Zusammenhang mit der gesellschaftlichen Thematisierung digitaler und interaktiver Medien und des damit verbundenen Wandels hin zu einer Informationsgesellschaft" (S. 9).

Im theoretischen Teil werden Begriffe wie Mediengeneration, Netzgeneration oder Generation@u. a. (S.13) im Hinblick auf die gesellschaftliche Konstruktion von Jugend und Generationsgestalten analysiert. Dieser Anspruch ist insofern spannend, weil der Autor mit seinem Titel „Die Netzgeneration“ sich selbst auf diesen Diskurs bezieht und damit ein Teil desselben wird. Ist sich der Autor dieser gewollten oder ungewollten Beteiligung an der Debatte über Generation und Jugend bewusst?

Zunächst charakterisiert der Autor die Beiträge der anderen Beteiligten an diesem Diskurs. Kritisch beschreibt er die dazugehörigen „Metaphernfelder“, „Motivationslagen“, „Begriffsfetische“ und das „Leitbildpotenzial“, die allesamt in ihren verschiedenen Formen dem „Überzeugungsdiskurs“ zur Ausformung und Diffusion bestehender und zukünftiger Technologien zu Grunde liegen und ihn beeinflussen. Hier zeige sich eine Fixierung zuerst auf das „Nutzungsverhalten der Anwender“, während die „Materialität“ der Medien weniger Aufmerksamkeit bekäme. Hebecker setzt sich inhaltsanalytisch mit der Rhetorik und den Mustern sowohl von wichtigen Printmedien (Wochenmagazine u. a) als auch von anderen „Meinungsführern“ auseinander. Deutlich wird, das fast gleichzeitig eine Reihe von Diskurstypen und -stilen nebeneinander existieren - populärwissenschaftliche, mediale, Szenereportagen, Generationsreportagen, „Selbstdeutungen einer Medienkohorte", 64-er Generation. Diese Diskurse, so der Autor, sind Teil einer „Risikokommunikation“ in der das Konzept der Generationen dazu dient, das Eigene 
und das Fremde zu formulieren und sich in diesen Diskursen jeweils zu positionieren - z. B. als „Integrierte“ oder als „Apokalyptiker“.

Der Eindruck beim Lesen dieser Arbeit ist widersprüchlich. Einerseits werden im 6. Kapitel eine Reihe von ,Typisierungen 'von Jugend in der Informationsgesellschaft beschrieben, die selbst ein Stück weit eben diese Muster wieder bestätigen und zugleich in ihrer begrenzten Gültigkeit demonstrieren. An dieser Stelle macht sich störend bemerkbar, dass der Bezug $\mathrm{zu}$ den Lebenswelten der verschiedenen $\mathrm{Ju}-$ gendkohorten blass bleibt. Die zu Grunde liegende Empirie in Form von Desideraten der Medienforschung und der damit verbundenen Interpretationen leisten diesen Bezug nur abstrakt. Das ist umso bedauerlicher, weil das Generationenkonzept von Manheim auch als Aufforderung verstanden werden kann, z. B. die "Gestaltungsintentionen" (Manheim) innerhalb einer Generation zu untersuchen, um die Signale des sozialen und kulturellen Wandels zu erkennen.

Die hier geleistete ausführliche Analyse der Diskurstypen zum Thema Jugend und Medien führt zu einem Paradoxon: Die Analyse der mediatisierten, publizierten Zeugnisse über die "Jugend" nehmen einen großen Raum ein, sie dominieren. Andererseits ist es eine Aufgabe der Sozialforschung, das Spannungsverhältnis zwischen den gesellschaftlich konstruierten Bildern über Jugend und ihrer begrenzten Gültigkeit zur Beschreibung und zum Verständnis der vielschichtigen Lebensbedingungen und den subjektiven Befindlichkeiten von Jugend anzusprechen. Es geht hier um eine Vermittlungsfunktion der Sozialwissenschaften: Beide - theoretische Konzepte und Desiderate der empirischen Forschung - bedürfen der kritischen Überprüfung und der Bewertung.

Bezogen auf das Thema „Netzgeneration Jugend in der Informationsgesellschaft" stellt sich die Frage, warum beim Lesen der Eindruck entsteht, dass die Ergebnisse der empirischen Jugendforschung relativ peripher aufgegriffen werden. Dieser Eindruck wird gestärkt, wenn die Ergebnisse der Peerforschung z. B. einen Zusammenhang zwischen der Ausdifferenzierung von Freundschaften und der Qualität der verschiedenen Einzelmedien aufzeigen. Die verschiedenen jugendlichen Altersgruppen entwickeln unterschiedliche Umgangsstile mit den Medien - das zeigt sich z. B. beim Internet (Szenen, Musikstile, Communities u. a.). Die
These der thematischen Auswahl der Medien hier und die These vom Umgang mit dem Medienmix dort - es scheint, dass die Aufmerksamkeit für die Diversifikation der Medien und die Ausdifferenzierung der Jugend und ihrer verschiedenen Teilgruppen und kulturellen Praxen miteinander korrespondieren. Die Analyse der verschiedenen Diskurstypen mit ihren medial verstärkten und von diesen $\mathrm{z}$. T. selbst konstruierten Typisierungen ist so gesehen hilfreich, weil damit zugleich deren Möglichkeiten und Grenzen aufzeigt werden können. Aber dieser Zugang zum Thema reicht nicht aus, wenn man mehr über die Besonderheiten der Netzgeneration erfahren will.

Auf der anderen Seite gelingt es der Untersuchung, das Augenmerk auf die impliziten Stereotypen und Wertmuster zu lenken, die hinter den gängigen Mustern und Begriffen der öffentlichen Debatte über Medien und Jugend stehen. Die Interpretationen und Kriterien für Hebeckers Verständnis von "Netzgeneration“" (z. B. als „Integration kultureller Gegensätze“, "als gesellschaftliche Regelung von Wissen") sind sehr anregend, um sie als Grundlage für den Diskurs über die Generationen und die Medien aufzugreifen und weiterzutragen.

Ekkehard Sander

\section{Bernd W. Wirtz}

\section{Medien- und Internetmanagement}

2. voll. überarb. und erw. Auflage

Wiesbaden: Gabler, 2001. - $571 \mathrm{~S}$

ISBN 3-409-21661-8

Bereits nach einem Jahr erscheint die 2. Auflage von Bernd W. Wirtz' Band „Medien- und Internetmanagement". Es handelt sich dabei um ein Lehrbuch, das eine systematische Aufarbeitung der unterschiedlichen Aspekte von Medienmanagement bietet. Dabei nimmt Bernd W. Wirtz klar und explizit eine betriebswirtschaftliche Perspektive ein und grenzt sich von kommunikationswissenschaftlichen und volkswirtschaftlichen Betrachtungen ab (S. 5), wiewohl er Medienmanagement als „Nachbardisziplin der Medienwissenschaften“ (S. 8) sieht. Entsprechend stützt er sich auf die Ansätze der klassischen Betriebswirtschaftslehre, was sich auch an der verwendeten Literatur zeigt, und konzipiert Medien- und Internetmanagement als einen neuen und zunehmend 